\title{
Stability analysis of a laser with two modulated saturable absorbers
}

\author{
Oscar Andres Naranjo-Montoya \\ Centro de Investigaciones en Óptica (CIO), Loma del Bosque 115, Lomas del Campestre, León, Guanajuato, CP 37150, Mexico
}

Received 27 April 2015; accepted 21 September 2015

Available online 29 November 2015

\begin{abstract}
The stability analysis of a model for a laser system with two modulated saturable absorbers, each one modulated at a different frequency, is performed. The model is based on four equations describing the temporal evolution of the photon flux, the population inversion in the active media, and the saturation coefficients of each saturable absorber. The system dynamics is discussed in order to find stable system control regions.

All Rights Reserved (c) 2015 Universidad Nacional Autónoma de México, Centro de Ciencias Aplicadas y Desarrollo Tecnológico. This is an open access item distributed under the Creative Commons CC License BY-NC-ND 4.0.
\end{abstract}

Keywords: Stability analysis; Laser system; Modulated saturable absorbers

\section{Introduction}

The dynamics of a laser system with two saturable absorbers (see Fig. 1) can be described by a model based on the Statz-DeMars equations, which originally were developed to describe oscillations in a Maser (Statz \& DeMars, 1960). This model has undergone many modifications to be adopted for laser systems (Tarassov, 1985; Tang \& Statz, 1963; Thompson \& Malacara, 2001). For a complete phenomenological description of a laser with two saturable absorbers, only four equations are needed: the photon-flux equation, an equation for the population inversion density in the active medium and two saturable population inversion equations that give the saturation coefficient for each saturable absorber (Wilson \& Aboites, 2013). Therefore, the Statz-DeMars equations for a three level laser system with two saturable absorbers without modulation are written as follows:

$$
\begin{aligned}
& \frac{d S}{d t}=\Gamma \nu \sigma N S-\Gamma \nu \frac{L_{\alpha_{1}}}{L_{m}} k_{\alpha_{1}} S-\Gamma \nu \frac{L_{\alpha_{2}}}{L_{m}} k_{\alpha_{2}} S-\frac{1}{T} S \\
& \frac{d N}{d t}=-\beta \frac{\sigma}{\hbar \omega} N S+\frac{N_{0}-N}{\tau} \\
& \frac{d k_{\alpha_{1}}}{d t}=-\frac{2 \sigma_{\alpha_{1}} k_{\alpha_{1}} S}{\hbar \omega}+\frac{k_{0 \alpha_{1}}-k_{\alpha_{1}}}{\tau_{\alpha_{1}}} \\
& \frac{d k_{\alpha_{2}}}{d t}=-\frac{2 \sigma_{\alpha_{2}} k_{\alpha_{2}} S}{\hbar \omega}+\frac{k_{0 \alpha_{2}}-k_{\alpha_{2}}}{\tau_{\alpha_{2}}},
\end{aligned}
$$

where $S$ is the emitted photon density, $N$ is the population inversion of the active medium, $k_{\alpha_{1}}$ and $k_{\alpha_{2}}$ are the resonant absorptions of the saturable absorbers 1 and 2 respectively, $\sigma_{\alpha_{1}}$ and $\sigma_{\alpha_{2}}$ are the saturable absorbers cross-sections, and $N_{\alpha_{1}}$ and $N_{\alpha_{2}}$ are the population inversions of the saturable absorbers $\left(k_{\alpha_{1}}=-\sigma_{\alpha_{1}} N_{\alpha_{1}}\right.$ and $\left.k_{\alpha_{2}}=-\sigma_{\alpha_{2}} N_{\alpha_{2}}\right) . \Gamma, \nu, \sigma$ and $T$ stand, respectively, for cavity filling coefficient, optical frequency, active medium cross-section and photon lifetime in the cavity; $\beta$ is the coefficient which accounts for the difference in population inversion coursed by lasing; $L_{m}, L_{\alpha_{1}}$ and $L_{\alpha_{2}}$ are, respectively, the active medium and the saturable absorbers lengths; $k_{0 \alpha_{1}}$ and $k_{0 \alpha_{2}}$ are the linear resonant saturable absorbers absorption coefficients without lasing; $N_{0}$ is

E-mail address: oscarnaranjo@ cio.mx

Peer Review under the responsibility of Universidad Nacional Autónoma de México. 


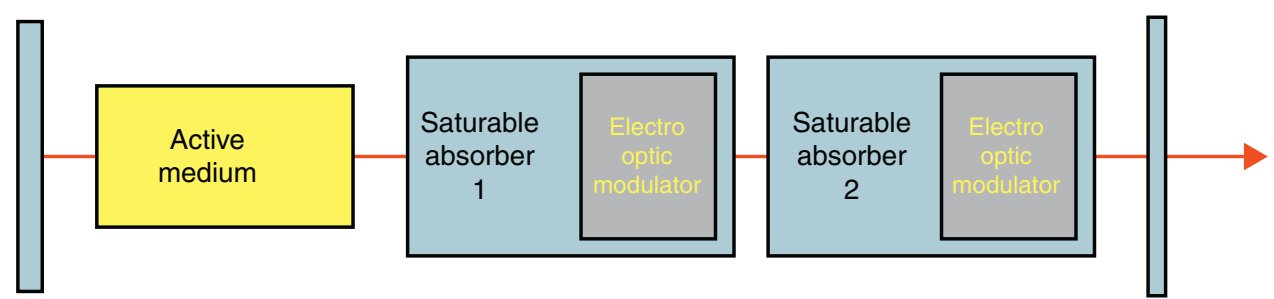

Fig. 1. Three-level laser system with two saturable absorbers with electro-optic modulators.

the population inversion in the active medium without radiation; $\tau, \tau_{\alpha_{1}}$ and $\tau_{\alpha_{2}}$ stand for relaxation time in the active medium and in the saturable absorbers, respectively; finally, $\hbar \omega$ is the photon energy (Aboites \& Ramírez, 1989).

Assuming that the two saturable absorbers have equal relaxation times $\left(\tau_{\alpha_{1}}=\tau_{\alpha_{2}}=\tau_{\alpha}\right)$, and defining the next adimensional parameters and variables: $t^{\prime}=t / \tau, G=\tau / t, \delta=\tau / \tau_{\alpha}, \rho_{1}=2 \sigma_{\alpha_{1}} / \beta \sigma, \rho_{2}=2 \sigma_{\alpha_{2}} / \beta \sigma, \alpha=\Gamma \nu \sigma T N$ and $\alpha_{\alpha_{1}}=-\Gamma \nu T k_{0 \alpha_{1}} L_{\alpha_{1}} / L_{m}=$ $-\Gamma \nu T \sigma_{\alpha_{1}} n_{\alpha_{1}} / L_{m}, \alpha_{\alpha_{2}}=-\Gamma \nu T k_{0 \alpha_{2}} L_{\alpha_{2}} / L_{m}=-\Gamma \nu T \sigma_{\alpha_{2}} n_{\alpha_{2}} / L_{m} ; n\left(t^{\prime}\right)=\Gamma \nu \sigma T N\left(t^{\prime}\right), n_{\alpha_{1}}\left(t^{\prime}\right)=-\Gamma \nu T k_{\alpha_{1}}\left(t^{\prime}\right) L_{\alpha_{1}} / L_{m}, n_{\alpha_{1}}\left(t^{\prime}\right)=$ $-\Gamma \nu T k_{\alpha_{2}}\left(t^{\prime}\right) L_{\alpha_{2}} / L_{m}$ and $n\left(t^{\prime}\right)=\beta B \tau S\left(t^{\prime}\right) / \nu=\beta \sigma \tau S\left(t^{\prime}\right) / \hbar \omega$, the above system can be rewritten as:

$$
\begin{aligned}
& \frac{d m}{d t^{\prime}}=G m\left(n+n_{\alpha_{1}}+n_{\alpha_{2}}-1\right) \\
& \frac{d n}{d t^{\prime}}=\alpha-n(m+1) \\
& \frac{d n_{\alpha_{1}}}{d t^{\prime}}=\delta \alpha_{\alpha_{1}}-n_{\alpha_{1}}\left(\rho_{1} m+\delta\right) \\
& \frac{d n_{\alpha_{2}}}{d t^{\prime}}=\delta \alpha_{\alpha_{2}}-n_{\alpha_{2}}\left(\rho_{2} m+\delta\right) .
\end{aligned}
$$

All the parameters used to define the saturable absorbers are fixed, except for $\alpha_{\alpha_{1}}$ and $\alpha_{\alpha_{2}}$, which include a measure of the active center absorbent density; for this reason, $\alpha_{\alpha_{1}}$ and $\alpha_{\alpha_{2}}$ are used as the saturable absorber identifying parameters. Adding an external linear sinusoidal modulation (e.g. using an Electro Optic Modulator (EOM)) directly into the saturable absorbers through their main parameter (i.e. $\alpha_{\alpha_{1}}$ and $\alpha_{\alpha_{2}}$ ), the last two above equations may be transformed into

$$
\begin{aligned}
\frac{d n_{\alpha_{1}}}{d t^{\prime}} & =\delta \alpha_{\alpha_{1}}\left[\frac{1+\cos \left(\omega_{c_{1}} t\right)}{2}\right]-n_{\alpha_{1}}\left(\rho_{1} m+\delta\right) \\
\frac{d n_{\alpha_{2}}}{d t^{\prime}} & =\delta \alpha_{\alpha_{2}}\left[\frac{1+\cos \left(\omega_{c_{2}} t\right)}{2}\right]-n_{\alpha_{2}}\left(\rho_{2} m+\delta\right),
\end{aligned}
$$

where $\omega_{c_{1}}$ and $\omega_{c_{2}}$ stand for the external modulation frequencies applied to the EOM. These four differential equations compose the working system. It must be noted that in absence of a modulation frequency applied to the EOM, $\omega_{c_{1}}$ and $\omega_{c_{2}}$, the system returns to Eq. (2), i.e. rate equations for a laser with two passive saturable absorbers (Wilson, Aboites, Pisarchik, Pinto, \& Barmenkov, 2011).

\section{Linear Stability Analysis}

Linear Stability Analysis is used to understand the system dynamics (Tabor, 1989; Braun, 1992). The analysis is based on the linear disturbance equations; these equations are derived from the original equations (Pinto Robledo et al., 2012). As it is well known, the method consists in linearizing the describing equations, obtaining the initial state condition (i.e. when the derivatives are zero), expanding the system about the initial state condition, constructing the Jacobian matrix, and finding the eigenvectors and eigenvalues with a determinant equal to zero. This gives, as a result, the fixed points of the equation system, which must be analyzed in order to know what type of points there are (i.e. fixed, source, saddle, etc.) (Wilson, Aboites, Pisarchik, Ruiz-Oliveras, \& Taki, 2011; Wilson, Aboites, Pisarchik, Pinto, \& Taki, 2011). The equations of interest are Eqs. (2) and (3); these equations are non-autonomous due 
to the explicit time-dependence found in the cosine of the third and fourth expressions. To be able to perform the Linear Stability Analysis, those dependences must be eliminated; to do that, variable changes must be applied, yielding, the next equation system:

$$
\begin{aligned}
& \frac{d m}{d t^{\prime}}=G m\left(n+n_{\alpha_{1}}+n_{\alpha_{2}}-1\right) \\
& \frac{d n}{d t^{\prime}}=\alpha-n(m+1) \\
& \frac{d n_{\alpha_{1}}}{d t^{\prime}}=\delta \alpha_{\alpha_{1}}\left[\frac{1+\cos \left(x_{1}\right)}{2}\right]-n_{\alpha_{1}}\left(\rho_{1} m+\delta\right) \\
& \frac{d n_{\alpha_{2}}}{d t^{\prime}}=\delta \alpha_{\alpha_{2}}\left[\frac{1+\cos \left(x_{2}\right)}{2}\right]-n_{\alpha_{2}}\left(\rho_{2} m+\delta\right), \\
& \frac{d x_{1}}{d t}=\omega_{c_{1}} \\
& \frac{d x_{2}}{d t}=\omega_{c_{2}}
\end{aligned}
$$

Naming Eq. (4) as:

$$
\begin{aligned}
& f\left(m, n, n_{\alpha_{1}}, n_{\alpha_{2}}, x_{1}, x_{2}\right)=\frac{d m}{d t^{\prime}} \\
& g\left(m, n, n_{\alpha_{1}}, n_{\alpha_{2}}, x_{1}, x_{2}\right)=\frac{d n}{d t^{\prime}} \\
& h\left(m, n, n_{\alpha_{1}}, n_{\alpha_{2}}, x_{1}, x_{2}\right)=\frac{d n_{\alpha_{1}}}{d t^{\prime}} \\
& j\left(m, n, n_{\alpha_{1}}, n_{\alpha_{2}}, x_{1}, x_{2}\right)=\frac{d n_{\alpha_{2}}}{d t^{\prime}} \\
& l\left(m, n, n_{\alpha_{1}}, n_{\alpha_{2}}, x_{1}, x_{2}\right)=\frac{d x_{1}}{d t} \\
& q\left(m, n, n_{\alpha_{1}}, n_{\alpha_{2}}, x_{1}, x_{2}\right)=\frac{d x_{2}}{d t},
\end{aligned}
$$

and assuming that $\left(m^{*}, n^{*}, n_{\alpha_{1}}{ }^{*}, n_{\alpha_{2}}{ }^{*}, x_{1}^{*}, x_{2}^{*}\right)$ is the steady state, that is, $f\left(m^{*}, n^{*}, n_{\alpha_{1}}^{*}, n_{\alpha_{2}}^{*}, x_{1}^{*}, x_{2}^{*}\right)=0$, $g\left(m^{*}, n^{*}, n_{\alpha_{1}}^{*}, n_{\alpha_{2}}^{*}, x_{1}^{*}, x_{2}^{*}\right)=0, h\left(m^{*}, n^{*}, n_{\alpha_{1}}^{*}, n_{\alpha_{2}}^{*}, x_{1}^{*}, x_{2}^{*}\right)=0, \quad j\left(m^{*}, n^{*}, n_{\alpha_{1}}^{*}, n_{\alpha_{2}}^{*}, x_{1}^{*}, x_{2}^{*}\right)=0, l\left(m^{*}, n^{*}, n_{\alpha_{1}}^{*}, n_{\alpha_{2}}^{*}, x_{1}^{*}, x_{2}^{*}\right)=0$, and $q\left(m^{*}, n^{*}, n_{\alpha_{1}}^{*}, n_{\alpha_{2}}^{*}, x_{1}^{*}, x_{2}^{*}\right)=0$, then in order to find whether the steady state is stable or unstable, a small perturbation (represented by the subscript $p$ ) must be added to it,

$$
\begin{aligned}
& f=f^{*}+f_{p} \\
& g=g^{*}+g_{p} \\
& h=h^{*}+h_{p} \\
& j=j^{*}+j_{p} \\
& l=l^{*}+l_{p} \\
& q=q^{*}+q_{p},
\end{aligned}
$$

With $\left(f_{p}, g_{p}, h_{p}, j_{p}, l_{p}, q_{p}\right) \ll 1$

Now, the main question for practical purposes is whether will the perturbations grow (steady state unstable) or decay (steady state stable). To be able to observe whether the perturbations grow or decay, the perturbation derivatives must be found (Wilson, Aboites, Pisarchik, et al., 2011).

$$
\begin{aligned}
\frac{d f_{p}}{d t}= & f\left(m^{*}, n^{*}, n_{a_{1}}^{*}, n_{a_{2}}^{*}, x_{1}^{*}, x_{2}^{*}\right)+\frac{\delta}{\delta m} f\left(m^{*}, n^{*}, n_{a_{1}}^{*}, n_{a_{2}}^{*}, x_{1}^{*}, x_{2}^{*}\right) f_{p}+\frac{\delta}{\delta n} f\left(m^{*}, n^{*}, n_{a_{1}}^{*}, n_{a_{2}}^{*}, x_{1}^{*}, x_{2}^{*}\right) g_{p} \\
& +\frac{\delta}{\delta n_{a_{1}}} f\left(m^{*}, n^{*}, n_{a_{1}}^{*}, n_{a_{2}}^{*}, x_{1}^{*}, x_{2}^{*}\right) h_{p}+\frac{\delta}{\delta n_{a_{2}}} f\left(m^{*}, n^{*}, n_{a_{1}}^{*}, n_{a_{2}}^{*}, x_{1}^{*}, x_{2}^{*}\right) j_{p}+\frac{\delta}{\delta x_{1}} f\left(m^{*}, n^{*}, n_{a_{1}}^{*}, n_{a_{2}}^{*}, x_{1}^{*}, x_{2}^{*}\right) l_{p} \\
& +\frac{\delta}{\delta x_{2}} f\left(m^{*}, n^{*}, n_{a_{1}}^{*}, n_{a_{2}}^{*}, x_{1}^{*}, x_{2}^{*}\right) q_{p}+\text { high order terms. }
\end{aligned}
$$


Following the Taylor series expansion shown in Eq. (7) the derivatives for each perturbation are:

$$
\begin{aligned}
\frac{d f_{p}}{d t} & =\frac{\delta}{\delta m} f \cdot f_{p}+\frac{\delta}{\delta n} f \cdot g_{p}+\frac{\delta}{\delta n_{\alpha_{1}}} f \cdot h_{p}+\frac{\delta}{\delta n_{\alpha_{2}}} f \cdot j_{p}+\frac{\delta}{\delta x_{1}} f \cdot l_{p}+\frac{\delta}{\delta x_{2}} f \cdot q_{p} \\
\frac{d g_{p}}{d t} & =\frac{\delta}{\delta m} g \cdot f_{p}+\frac{\delta}{\delta n} g \cdot g_{p}+\frac{\delta}{\delta n_{\alpha_{1}}} g \cdot h_{p}+\frac{\delta}{\delta n_{\alpha_{2}}} g \cdot j_{p}+\frac{\delta}{\delta x_{1}} g \cdot l_{p}+\frac{\delta}{\delta x_{2}} g \cdot q_{p} \\
\frac{d h_{p}}{d t} & =\frac{\delta}{\delta m} h \cdot f_{p}+\frac{\delta}{\delta n} h \cdot g_{p}+\frac{\delta}{\delta n_{\alpha_{1}}} h \cdot h_{p}+\frac{\delta}{\delta n_{\alpha_{2}}} h \cdot j_{p}+\frac{\delta}{\delta x_{1}} h \cdot l_{p}+\frac{\delta}{\delta x_{2}} h \cdot q_{p} \\
\frac{d j_{p}}{d t} & =\frac{\delta}{\delta m} j \cdot f_{p}+\frac{\delta}{\delta n} j \cdot g_{p}+\frac{\delta}{\delta n_{\alpha_{1}}} j \cdot h_{p}+\frac{\delta}{\delta n_{\alpha_{2}}} j \cdot j_{p}+\frac{\delta}{\delta x_{1}} j \cdot l_{p}+\frac{\delta}{\delta x_{2}} j \cdot q_{p} \\
\frac{d l_{p}}{d t} & =\frac{\delta}{\delta m} l \cdot f_{p}+\frac{\delta}{\delta n} l \cdot g_{p}+\frac{\delta}{\delta n_{\alpha_{1}}} l \cdot h_{p}+\frac{\delta}{\delta n_{\alpha_{2}}} l \cdot j_{p}+\frac{\delta}{\delta x_{1}} l \cdot l_{p}+\frac{\delta}{\delta x_{2}} l \cdot q_{p} \\
\frac{d q_{p}}{d t} & =\frac{\delta}{\delta m} q \cdot f_{p}+\frac{\delta}{\delta n} q \cdot g_{p}+\frac{\delta}{\delta n_{\alpha_{1}}} q \cdot h_{p}+\frac{\delta}{\delta n_{\alpha_{2}}} q \cdot j_{p}+\frac{\delta}{\delta x_{1}} q \cdot l_{p}+\frac{\delta}{\delta x_{2}} q \cdot q_{p} .
\end{aligned}
$$

The system presented in Eq. (8) can be rewritten in the matrix form:

$$
\left(\begin{array}{c}
f_{p}^{\prime} \\
g_{p}^{\prime} \\
h_{p}^{\prime} \\
j_{p}^{\prime} \\
l_{p}^{\prime} \\
q_{p}^{\prime}
\end{array}\right)=\left(\begin{array}{cccccc}
\frac{\delta}{\delta m} f & \frac{\delta}{\delta n} f & \frac{\delta}{\delta n_{a_{1}}} f & \frac{\delta}{\delta n_{a 2}} f & \frac{\delta}{\delta x_{1}} f & \frac{\delta}{\delta x_{2}} f \\
\frac{\delta}{\delta m} g & \frac{\delta}{\delta n} g & \frac{\delta}{\delta n_{a_{1}}} g & \frac{\delta}{\delta n_{a 2}} g & \frac{\delta}{\delta x_{1}} g & \frac{\delta}{\delta x_{2}} g \\
\frac{\delta}{\delta m} h & \frac{\delta}{\delta n} h & \frac{\delta}{\delta n_{a_{1}}} h & \frac{\delta}{\delta n_{a 2}} h & \frac{\delta}{\delta x_{1}} h & \frac{\delta}{\delta x_{2}} h \\
\frac{\delta}{\delta m} j & \frac{\delta}{\delta n} j & \frac{\delta}{\delta n_{a_{1}}} j & \frac{\delta}{\delta n_{a 2}} j & \frac{\delta}{\delta x_{1}} j & \frac{\delta}{\delta x_{2}} j \\
\frac{\delta}{\delta m} l & \frac{\delta}{\delta n} l & \frac{\delta}{\delta n_{a_{1}}} l & \frac{\delta}{\delta n_{a 2}} l & \frac{\delta}{\delta x_{1}} l & \frac{\delta}{\delta x_{2}} l \\
\frac{\delta}{\delta m} q & \frac{\delta}{\delta n} q & \frac{\delta}{\delta n_{a_{1}}} q & \frac{\delta}{\delta n_{a 2}} q & \frac{\delta}{\delta x_{1}} q & \frac{\delta}{\delta x_{2}} q
\end{array}\right)\left(\begin{array}{c}
f_{p} \\
g_{p} \\
h_{p} \\
j_{p} \\
l_{p} \\
q_{p}
\end{array}\right)=j\left(\begin{array}{c}
f_{p} \\
g_{p} \\
h_{p} \\
j_{p} \\
l_{p} \\
q_{p}
\end{array}\right),
$$

where $j$ denotes the Jacobian matrix of the original system at the steady state. Substituting the values in the Jacobian function, the next matrix is obtained:

$$
\left(\begin{array}{cccccc}
G\left(n+n_{\alpha_{1}}+n_{\alpha_{2}}-1\right) & G m & G m & G m & 0 & 0 \\
-n & -m-1 & 0 & 0 & 0 & 0 \\
-n_{\alpha_{1}} \rho_{1} & 0 & -\rho_{1} m-\delta & 0 & -\frac{\delta \alpha_{\alpha_{1}}}{2} \sin \left(x_{1}\right) & 0 \\
-n_{\alpha_{2}} \rho_{2} & 0 & 0 & -\rho_{2} m-\delta & 0 & -\frac{\delta \alpha_{\alpha_{2}}}{2} \sin \left(x_{2}\right) \\
0 & 0 & 0 & 0 & 0 & 0 \\
0 & 0 & 0 & 0 & 0 & 0
\end{array}\right) .
$$

The next step is to find the eigenvectors and eigenvalues of the system $(j-\lambda I) \partial_{p}=0$; so, the determinant would be:

$$
|j-\lambda I|=0 .
$$

The matrix for the former equation is:

$$
\left(\begin{array}{cccccc}
G\left(n+n_{\alpha_{1}}+n_{\alpha_{2}}-1\right)-\lambda & G m & G m & G m & 0 & 0 \\
-n & -m-1-\lambda & 0 & 0 & 0 & 0 \\
-n_{\alpha_{1}} \rho_{1} & 0 & -\rho_{1} m-\delta-\lambda & 0 & -\frac{\delta \alpha_{\alpha_{1}}}{2} \sin \left(x_{1}\right) & 0 \\
-n_{\alpha_{2}} \rho_{2} & 0 & 0 & -\rho_{2} m-\delta-\lambda & 0 & -\frac{\delta \alpha_{\alpha_{2}}}{2} \sin \left(x_{2}\right) \\
0 & 0 & 0 & 0 & -\lambda & 0 \\
0 & 0 & 0 & 0 & 0 & -\lambda
\end{array}\right)=0 .
$$




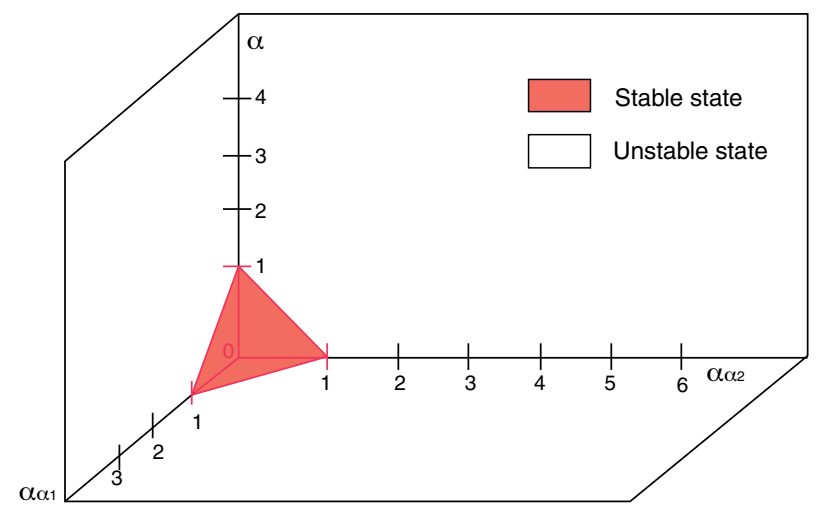

Fig. 2. Stability condition given by the relation between $\alpha_{\alpha_{1}}, \alpha_{\alpha_{2}}$ and $\alpha$.

The solutions for the perturbed steady-state of the original system are:

$$
\begin{aligned}
& m^{s}=0 \\
& n^{s}=\alpha \\
& x_{1}^{s}=0 \\
& x_{2}^{s}=0 \\
& n_{\alpha_{1}}^{s}=\alpha_{\alpha_{1}} \\
& n_{\alpha_{2}}^{s}=\alpha_{\alpha_{2}} .
\end{aligned}
$$

Substituting the former solutions in Eq. (12), the matrix transforms into:

$$
\left(\begin{array}{cccccc}
G\left(\alpha+\alpha_{\alpha_{1}}+\alpha_{\alpha_{2}}-1\right)-\lambda & 0 & 0 & 0 & 0 & 0 \\
-\alpha & -1-\lambda & 0 & 0 & 0 & 0 \\
-\alpha_{\alpha_{1}} \rho_{1} & 0 & -\delta-\lambda & 0 & 0 & 0 \\
-\alpha_{\alpha_{2}} \rho_{2} & 0 & 0 & -\delta-\lambda & 0 & 0 \\
0 & 0 & 0 & 0 & -\lambda & 0 \\
0 & 0 & 0 & 0 & 0 & -\lambda
\end{array}\right)=0 .
$$

Matrix (14) has the following characteristic equation:

$$
\left[G\left(\alpha+\alpha_{\alpha_{1}}+\alpha_{\alpha_{2}}-1\right)-\lambda\right][-1-\lambda][2(-\delta-\lambda)][-2 \lambda]=0,
$$

where $\lambda_{1}=G\left(\alpha+\alpha_{\alpha_{1}}+\alpha_{\alpha_{2}}-1\right), \lambda_{2}=-1, \lambda_{3}=-\delta, \lambda_{4}=-\delta, \lambda_{5}=0$ and $\lambda_{6}=0$ are eigenvalues which are all real; $\lambda_{2}, \lambda_{3}$ and $\lambda_{4}$ are always negative (i.e. the perturbation will decay), and $\lambda_{5}$ and $\lambda_{6}$ are critically stable. Therefore, the stability condition is defined only by the sign of $\lambda_{1}$, i.e. the fixed point is a source when $\left(\alpha_{\alpha_{1}}+\alpha_{\alpha_{2}}+\alpha\right)>1$, as shown in Figure 2 .

\section{Conclusions}

The stability analysis of the model for a laser system with two modulated saturable absorbers is performed. The model is based on four equations describing the temporal evolution of the photon flux, the population inversion in the active media, and the saturation coefficients of the two saturable absorbers. The stability condition of the systems is found to depend only on the sign of the first eigenvalue of the system $\lambda$. As it is graphically shown, the stability conditions are given by the relation between $\alpha, \alpha_{\alpha_{1}}$ and $\alpha_{\alpha_{2}}$.

\section{Conflict of interest}

The authors have no conflicts of interest to declare. 


\section{References}

Aboites, V., \& Ramírez, S. (1989). Stability of the Statz-DeMars equation in the description of a laser. Revista Mexicana de Física, 35(2), 326-333.

Braun, M. (1992). Differential equations and their applications: An introduction to applied mathematics. Springer.

Pinto Robledo, V. J., Lopez, G., Espinosa, Y. M., Pisarchik, A. N., Jaimes Reátegui, R., \& Aboites, V. (2012). Experimental study of the dynamics of a diode-pumped $\mathrm{Nd}: \mathrm{YVO}_{4}$ laser under periodic modulation of losses. Revista Mexicana de Física E, 58(2), 150-155.

Statz, H., \& DeMars, G. (1960). Transients and oscillation pulses in masers. In C. Townes (Ed.), Quantum electronics (pp. 530-537). New York: Columbia University Press.

Tabor, M. (1989). Linear stability analysis. In Chaos and integrability in nonlinear dynamics: An introduction. pp. 20-31. New York: Wiley.

Tang, C. L., \& Statz, H. (1963). Spectral output and spiking behavior of solid-state lasers. Journal of Applied Physics, 34(8), $2289-2295$.

Tarassov, L. (1985). Physique des Processus dans les Générateurs de Rayonnement Optique Cohérent. Moscow: MIR.

Thompson, B. J., \& Malacara, D. (2001). Handbook of optical engineering. pp. 978. CRC Press.

Wilson, M., \& Aboites, V. (2013). Stability analysis of a laser with a modulated saturable absorber. International Journal of Pure and Applied Mathematics, 82(4), 623-629.

Wilson, M., Aboites, V., Pisarchik, A. N., Pinto, V., \& Taki, M. (2011). Generation of cnoidal waves by a laser system with a controllable saturable absorber. Optics Express, 19(15), 14210-14216.

Wilson, M., Aboites, V., Pisarchik, A. N., Ruiz-Oliveras, F., \& Taki, M. (2011). Stable cnoidal wave formation in an erbium-doped fiber laser. Applied Physics Express, 4(11), 112701.

Wilson, M., Aboites, V., Pisarchik, A., Pinto, V., \& Barmenkov, Y. (2011). Controlling a laser output through an active saturable absorber. Revista Mexicana de Física, 57(3), 250-254. 